\title{
The prevalence of halitosis (oral malodor) and associated factors among dental students and interns, Lahore, Pakistan
}

\author{
Muhammad Ashraf Nazir ${ }^{1}$, Khalid Almas ${ }^{1}$, Muhammad Irfan Majeed ${ }^{2}$
}

Correspondence: Dr. Muhammad Ashraf Nazir

Email:manazir@uod.edu.sa
'Department of Preventive Dental Sciences, University of Dammam College of Dentistry, Dammam, KSA, ${ }^{2}$ Department of Prosthetics, College of Dentistry, King Khalid University, Abha, KSA

\section{ABSTRACT}

Objective: To evaluate the prevalence of halitosis and the factors associated with it among dental students and interns in Lahore, Pakistan. Methods: A cross-sectional study design was chosen, and a sample of dental students and interns was collected from seven dental colleges in Lahore, Pakistan. A total of 833 participants were approached in person as convenient sample population. A self-reported questionnaire was administered and informed consent was obtained. The associations between oral malodor and different variables of the study were explored using analytical statistics (Chi-square test and logistic regression analysis). Statistical significance was determined using a 95\% confidence interval (CI). Results: Six hundred and fifteen participants (aged 19-27 years) completed the survey with a response rate of $73.8 \%$. The prevalence of self-reported halitosis was $75.1 \%$. More female (51.4\%) than male students (23.7\%) reported oral malodor, and most participants (61\%) reported early morning halitosis. Thirteen percent of respondents had examination for oral malodor by a dentist and $37.6 \%$ treated the condition with self-medication. Binary logistic regression model showed that male gender (odds ratio $[\mathrm{OR}]=0.44, \mathrm{CI}=0.22-0.87$ ), daily use of dental floss $(\mathrm{OR}=0.28, \mathrm{CI}=0.13-0.58)$, and drinking tea with mint $(\mathrm{OR}=0.44, \mathrm{CI}=0.22-0.89)$ were significantly associated with oral malodor. The participants with tongue coating had higher odds $(\mathrm{OR}=2.75, \mathrm{CI}=1.13-6.69)$ of having oral malodor than those without tongue coating, and the association was statistically significant. Conclusions: The study identified high prevalence of oral malodor among dental students and interns. They should receive appropriate diagnosis and management of the condition from dentist. The regular use of dental floss and removal of tongue coating can significantly reduce halitosis.

Key words: Dental students, halitosis, oral malodor, tongue coatings

\section{INTRODUCTION}

Halitosis, also known as oral malodor or bad breath, is a common oral condition characterized by unpleasant or offensive odor originating from the mouth of an individual and is noticed by others. ${ }^{[1,2]}$ The condition is caused by necrotic pulpal exposure, deep carious lesions, food impaction, oral infections, periodontal disease, faulty restorations, reduced salivary flow, smoking,

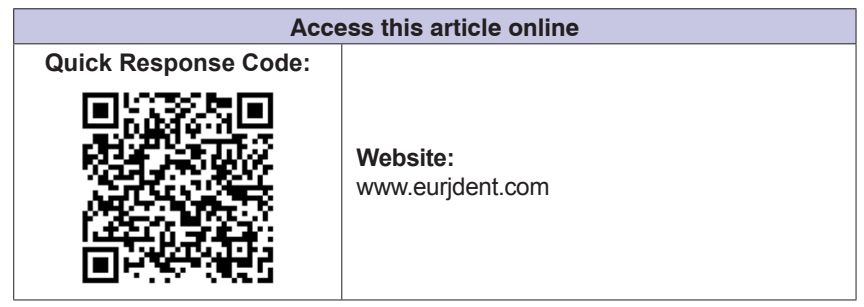

and poor oral hygiene practices including not brushing and flossing. ${ }^{[3-7]}$ Systemic diseases such as respiratory and gastrointestinal conditions, carcinomas, and certain medications can also contribute to oral malodor. ${ }^{[5]}$

Millions of people around the globe suffer from halitosis; however, some people believe that they have

This is an open access article distributed under the terms of the Creative Commons Attribution-NonCommercial-ShareAlike 3.0 License, which allows others to remix, tweak, and build upon the work non-commercially, as long as the author is credited and the new creations are licensed under the identical terms.

For reprints contact: reprints@medknow.com

How to cite this article: Nazir MA, Almas K, Majeed MI. The prevalence of halitosis (oral malodor) and associated factors among dental students and interns, Lahore, Pakistan. Eur J Dent 2017;11:480-5.

DOI: 10.4103/ejd.ejd_142_17 
a problem, but condition is clinically absent among them (pseudo halitosis) whereas others have halitosis but are unaware and do not accept the existence of the bad breath (denied halitosis) ${ }^{[8]}$ Literature shows that approximately $28 \%$ of patients complaining of halitosis were not found to have signs of the condition. ${ }^{[9]}$ On the other hand, oral malodor was perceived by $42 \%$ of respondents while it was detected clinically in $39.6 \%$ of them. ${ }^{[10]}$ Further, halitophobia is the term used for those subjects who have exaggerated fear of halitosis. ${ }^{[11]}$

The studies have documented a high prevalence of oral malodor in young, adult, and elderly populations in different regions of the world. Forty-two percent of Japanese school children reported that they had oral malodor. ${ }^{[10]}$ In a study of Korean adolescents, $23.6 \%$ of the samples were found to have subjective halitosis. ${ }^{[12]}$ The results of a study by Bornstein et al. identified 32\% of adults with halitosis in Switzerland. ${ }^{[13]}$ Organoleptic assessments conducted in Italian adult population revealed that $53.51 \%$ of subjects experienced oral malodor. ${ }^{[14]}$ Recently, AlSadhan reported the presence of self-perceived halitosis in $22.8 \%$ of study participants (aged 17-65 years) in Saudi Arabia. ${ }^{15]}$ The prevalence of self-reported halitosis was $28.3 \%$ in Turkish individuals aged $>55$ years. ${ }^{[16]}$ The prevalence rates range from $20 \%$ to $>50 \%$ of world population. ${ }^{[3]}$

It is important for dentists to understand self-perception about halitosis because the condition is related to self-image and psychopathological characteristics of the patients as they are the primary health-care providers managing the condition. ${ }^{[17]}$ It has been suggested that dentists should also be aware of cultural and social norms about halitosis so that they can provide optimal oral care to the patients to improve their quality of life.$^{[18]}$ An investigation of clinical observations suggests a link between halitosis and anxiety ${ }^{[19,20]}$ as a high concentration of sulfur compounds was found among anxious patients. ${ }^{[19]}$ Moreover, the patients with physiological halitosis also exhibit symptoms of depression. ${ }^{[21]}$

Only few studies investigated halitosis, oral hygiene practices, and smoking behaviors among dental students. ${ }^{[22-25]}$ These previous studies included small samples of students taken from one dental institution. Therefore, the objective of the study was to evaluate the prevalence of halitosis and the factors associated with it in a large sample of dental students and interns.

\section{METHODS}

\section{Participants}

A cross-sectional study design was chosen, and a sample population of dental students and interns was collected from seven dental colleges in Lahore, Pakistan. Initially, the consent to conduct the study was obtained from respective dental colleges, and a person was designated in each institution for distribution and collection of questionnaires. To ensure adequate sample of the study participants, the questionnaires were distributed among all the available and willing to participate students and interns in each dental college. A total of 833 participants were approached in person and invited to participate in the study. Nonprobability sampling method (convenience sampling technique) was used. The survey was conducted from June 2016 to September 2016. For those who agreed to participate in the study were provided with the details of the study including its objective and purpose and voluntary participation of respondents. The respondents provided informed consent.

\section{Study measures}

The questionnaire used in the present study was taken from a previous study conducted in similar settings and population. ${ }^{[25]}$ The questionnaire included information about age and gender of the respondents. The instrument ascertained the participants' responses about halitosis. They were asked to assess their breath on a visual analog scale (VAS) from 0 to 10 by encircling the appropriate number that corresponded to their severity of oral malodor. The 0 on VAS represented the absence of malodor while 10 meant maximum bad breath. The respondents provided information about visiting dentist/physician for consultation, treatment-seeking behaviors included self-medication, oral hygiene practices, smoking, dental caries, bleeding gums, dryness of mouth, and use of tea with mint.

\section{Statistical analysis}

SPSS (IBM Corp. Released 2013. IBM SPSS Statistics for Windows, version 22.0. IBM Corp., Armonk, NY, USA) was used for statistical analysis. Standard descriptive statistics were used to report characteristics and responses of study participants. The association between oral malodor and different variables of the study was explored using Chi-square test and binary logistic regression analysis. Statistical significance was determined using 95\% confidence interval. 


\section{RESULTS}

Six hundred and fifteen participants responded to the questionnaire (response rate $73.8 \%$ ). Females represented greater percentage $(68 \%)$ of the study sample. The age of respondents ranged from 19 to 27 years (mean 22.7, standard deviation [SD] \pm 5.9 ). The prevalence of self-reported halitosis was $75.1 \%$ as about one-quarter $(24.9 \%)$ of the participants marked 0 on the VAS scale [Table 1]. More female $(51.4 \%)$ than male subjects $(23.7 \%)$ reported oral malodor.

The majority of the respondents (75.1\%) were bothered with halitosis, and $76.6 \%$ believed that it was not normal to have oral malodor; however, only small proportion $(11.9 \%)$ considered that halitosis affected their quality of life. A considerable number $(n=231,37.6 \%)$ of them used self-medication to treat bad breath, and few participants consulted dentist and physician [Table 1]. Most participants (61\%) reported that they had oral malodor after waking up [Figure 1].

Cross-tabulation with Chi-square tests revealed that the participants who used dental floss daily were less likely to have oral malodor (unadjusted odds ratio $[\mathrm{OR}]=0.28$ ), and the relationship was statistically significant. Similarly, drinking tea with mint was significantly associated with lower odds of having oral malodor (unadjusted $\mathrm{OR}=0.53$ ) [Table 2]. In binary logistic regression model, male gender $(\mathrm{OR}=0.44)$, daily use of dental floss $(\mathrm{OR}=0.28)$, and drinking tea with mint $(\mathrm{OR}=0.44)$ were significantly associated with oral malodor after controlling for other variables of the study summarized in Table 3. The respondents

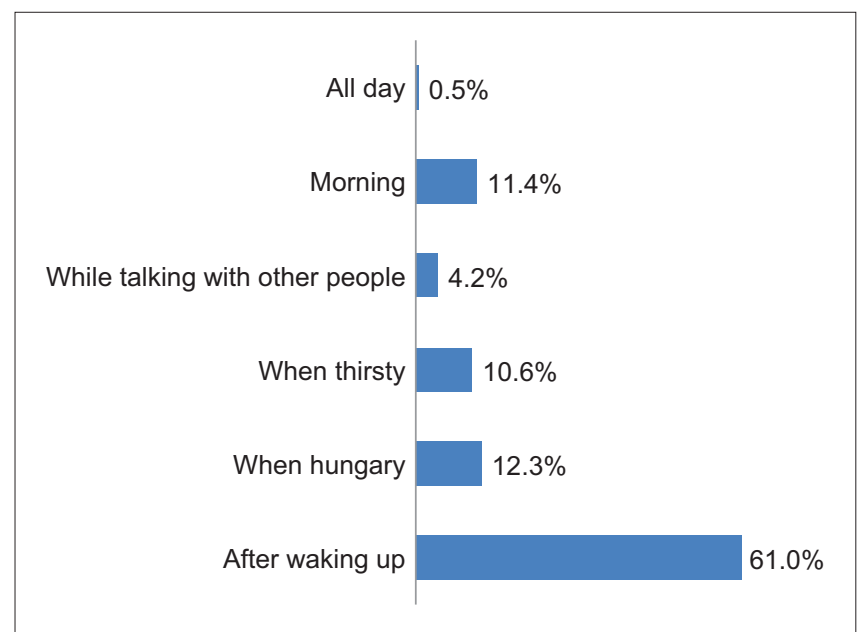

Figure 1: Oral malodor at different times of the day with tongue coating had higher odds $(\mathrm{OR}=2.75)$ of having oral malodor, and the association was statistically significant [Table 3].

\section{DISCUSSION}

The findings of this study indicate that the prevalence of oral malodor was high among study participants, and majority of them considered having the

\begin{tabular}{|c|c|}
\hline & $n(\%)$ \\
\hline \multicolumn{2}{|l|}{ Assessment of oral malodor using VAS scale } \\
\hline 0 & $153(24.9)$ \\
\hline 1 & $60(9.8)$ \\
\hline 2 & $91(14.8)$ \\
\hline 3 & $87(14.1)$ \\
\hline 4 & $61(9.9)$ \\
\hline 5 & $78(12.7)$ \\
\hline 6 & $37(6.0)$ \\
\hline 7 & $24(3.9)$ \\
\hline 8 & $12(2.0)$ \\
\hline 9 & $1(0.2)$ \\
\hline 10 & $11(1.8)$ \\
\hline \multicolumn{2}{|l|}{ Responses about oral malodor } \\
\hline \multicolumn{2}{|l|}{ Examination for oral malodor by } \\
\hline Dentist & $77(13.1)$ \\
\hline Physician & $38(7.4)$ \\
\hline \multicolumn{2}{|l|}{ Treatment received for oral malodor by } \\
\hline Dentist & $67(11.2)$ \\
\hline Physician & $23(4.5)$ \\
\hline Self-medication & $231(37.6)$ \\
\hline Traditional medication & $85(17)$ \\
\hline Oral malodor interfere with your social life & $73(11.9)$ \\
\hline Normal to have oral malodor & $144(23.4)$ \\
\hline Oral malodor bothers & $462(75.1)$ \\
\hline
\end{tabular}

\begin{tabular}{|c|c|c|}
\hline Variables & Unadjusted OR & $95 \% \mathrm{Cl}$ \\
\hline \multicolumn{3}{|l|}{ Gender } \\
\hline Male & 0.92 & $0.62-1.36$ \\
\hline \multicolumn{3}{|l|}{ Female } \\
\hline Regular tooth brushing & 2.26 & $0.89-5.73$ \\
\hline Daily use of dental floss & 0.28 & $0.19-0.42^{*}$ \\
\hline Daily use of mouthwash & 0.72 & $0.49-1.05$ \\
\hline Tooth decay & 1.03 & $0.71-1.52$ \\
\hline Bleeding gums & 0.82 & $0.54-1.25$ \\
\hline Dryness of mouth & 0.81 & $0.49-1.34$ \\
\hline Regular smoking & 1.14 & $0.65-1.99$ \\
\hline Drinking tea with mint regularly & 0.53 & $0.35-0.79^{*}$ \\
\hline Tongue coating & 1.23 & $0.65-2.34$ \\
\hline
\end{tabular}

*Statistically significant. OR: Odds ratio, $\mathrm{Cl}$ : Confidence interval 


\begin{tabular}{|c|c|c|}
\hline Variables & Adjusted OR & $95 \% \mathrm{Cl}$ \\
\hline \multicolumn{3}{|l|}{ Gender } \\
\hline Male & 0.44 & $0.22-0.87^{*}$ \\
\hline \multicolumn{3}{|l|}{ Female } \\
\hline Regular tooth brushing & 3.33 & $0.81-13.64$ \\
\hline Daily use of dental floss & 0.28 & $0.13-0.58^{*}$ \\
\hline Daily use of mouthwash & 1.18 & $0.58-2.37$ \\
\hline Tooth decay & 0.86 & $0.43-1.73$ \\
\hline Bleeding gums & 0.68 & $0.29-1.57$ \\
\hline Dryness of mouth & 0.57 & $0.22-1.49$ \\
\hline Regular smoking & 1.81 & $0.60-5.42$ \\
\hline Drinking tea with mint regularly & 0.44 & $0.22-0.89^{*}$ \\
\hline Tongue coating & 2.75 & $1.13-6.69^{*}$ \\
\hline
\end{tabular}

condition not normal and were bothered with it. Most respondents reported having early morning halitosis. Few students and interns consulted dentist or physician for diagnosis and treatment of oral malodor, and self-medical and traditional medicine were used by half of them. The results suggest a moderate association between tongue coating and halitosis. Male gender, use of dental floss, and drinking tea with mint were independently associated with lower odds of oral malodor.

In a study of 481 Saudi dental students, Almas et al. identified $44 \%$ of males and $32 \%$ of females with self-perception of oral malodor. ${ }^{[25]}$ Ashwath et al. ${ }^{[24]}$ found oral malodor in $44.1 \%$ of male and $45.32 \%$ of female Indian dental students. Similarly, Setia et al. .23] $^{[3}$ demonstrated that $40 \%$ of female compared with $58 \%$ of male dental students reported self-perceived halitosis in India. Nevertheless, the findings of present study revealed that higher percentage of female than male students reported oral malodor and there was a significant association between gender and halitosis. In binary logistic regression model, females were $56 \%$ more likely than males to have oral malodor. A recent study compared clinical with perceived oral malodor among Malaysian dental students and found that higher percentage of students $(52.7 \%)$ had clinical than $19 \%$ with self-perception of malodor. ${ }^{[22]}$ This underscores the importance of reporting self-perceived oral malodor in our study.

Few dental students and interns consulted a dentist or a physician for the diagnosis and treatment of halitosis and more than half used self-medication and traditional medicine in our study. Similar oral health-care seeking behaviors were observed in
Saudi dental students in a study by Almas et al. ${ }^{[25]}$ The authors reported that $8 \%$ of male and $3 \%$ of female students were diagnosed by a dentist, $12 \%$ of males and $26 \%$ of females treated themselves, and $15 \%$ used traditional medication. This reflects that students and interns avoid consulting dental care providers possibly because of social stigma attached with halitosis. ${ }^{[26]}$ As future dental professional, the students should understand clinical and social aspects of the condition and emphasize the role of dentist in preventing and controlling oral malodor.

It was found that significant relationship exists between tongue coating measured with tongue coating index and halitosis. ${ }^{[27]}$ Similarly, previous studies identified tongue coating as an etiological factor in the development of halitosis. ${ }^{[28-30]}$ The results of logistic regression analysis of a previous study found a strong relationship between clinical malodor and tongue coating; however, no such association was observed between self-reported tongue coating with oral malodor. ${ }^{[22]}$ Our study results showed that students with tongue coating were 2.75 times more likely to have malodor compared with those without tongue coating. Similar to our results, an association between tongue coating and self-perceived oral malodor $(\mathrm{OR}=3.53)$ was observed in Thai population. ${ }^{[31]}$ In addition, it was demonstrated that reduction in morning halitosis occurred after removing tongue coating. ${ }^{[32]}$

The production of volatile sulfur compounds resulting from the bacterial decomposition of organic substrate by Gram-negative anaerobes in periodontal sites and on the dorsum of tongue is considered the main cause of halitosis. ${ }^{[23,33]}$ Higher levels of sulfur compounds were observed among females compared with males and at the time of awakening than later in the morning. ${ }^{[34]}$ Our results indicate that most participants had early morning halitosis and these findings are in accordance with the results of previous studies. ${ }^{[23-25]}$

Our study confirmed that use of dental floss was associated with lower odds of having oral malodor after controlling for other factors. This agrees with the findings reported recently by AlSadhan ${ }^{[15]}$ who demonstrated statistically significant relationship between self-reported halitosis and use of dental floss. Faveri et al..$^{[32]}$ evaluated the effect of interdental flossing on oral malodor and found that there was a significantly lower concentration of sulfur compounds after using dental floss. Almas et al. showed that considerable percentage of dental 
students $($ male $=43.8 \%$, female $=37.2 \%$ ) consumed mint flavored tea. In our study, the participants who regularly took tea with mint had lower chances of having halitosis than those who did not consume tea with mint.

It has been shown that smoking, periodontal disease, dryness of mouth, and deep carious lesions can cause halitosis. ${ }^{[3,4]}$ Moreover, a previous similar study reported a significant association between oral malodor and dryness of mouth and smoking among dental students. ${ }^{[23]}$ On the contrary, our study results showed no significant relationship between oral malodor and dental caries, smoking, dryness of mouth, and bleeding gums. This could be because more female students reported oral malodor, and low prevalence of oral conditions is found in younger dental professional population.

The study identified several important factors associated with halitosis among dental students and interns, and the findings are of great importance as a representative sample was collected from seven dental colleges in Lahore. Nevertheless, given the large number of dental schools in Pakistan and the limitations of time and resources, it was not feasible to collect data from most dental institutions in the country. In addition, a cross-sectional study design has its weakness with regard to the investigation of causal inference. ${ }^{[35]}$ Therefore, randomized clinical trials should be conducted to evaluate if the use of dental floss and tea with mint reduce halitosis.

\section{CONCLUSION}

There is a high prevalence of oral malodor among dental students and interns. They should receive appropriate diagnosis and management of the condition from dentist. The use of dental floss can significantly reduce the prevalence of halitosis. Similarly, removal of tongue coating can result in the reduction of oral malodor. As future oral health care professionals, dental students and interns should regularly use dental floss and remove tongue coating to ensure high standards of dental care with pleasing breath when dealing patients and colleagues.

\section{Financial support and sponsorship}

Nil.

\section{Conflicts of interest}

There are no conflicts of interest.

\section{REFERENCES}

1. Rosenberg M. The science of bad breath. Sci Am 2002;286:72-9.

2. Kapoor U, Sharma G, Juneja M, Nagpal A. Halitosis: Current concepts on etiology, diagnosis and management. Eur J Dent 2016;10:292-300.

3. Cortelli JR, Barbosa MD, Westphal MA. Halitosis: A review of associated factors and therapeutic approach. Braz Oral Res 2008;22 Suppl 1:44-54.

4. Pratibha PK, Bhat KM, Bhat GS. Oral malodor: A review of the literature. J Dent Hyg 2006;80:8.

5. van den Broek AM, Feenstra L, de Baat C. A review of the current literature on aetiology and measurement methods of halitosis. J Dent 2007;35:627-35.

6. Al-Ansari JM, Boodai H, Al-Sumait N, Al-Khabbaz AK, Al-Shammari KF, Salako N. Factors associated with self-reported halitosis in Kuwaiti patients. J Dent 2006;34:444-9.

7. Nazir MA, Almas K. Awareness about the effects of tobacco consumption on oral health and the possibility of smoking behavior among male Saudi schoolchildren. Eur J Dent 2017;11:29-35.

8. Nardi GM, Forabosco A, Forabosco G, Musciotto A, Campisi G, Grandi T. Halitosis: A stomatological and psychological issue. Minerva Stomatol 2009;58:435-44

9. Bollen CM, Beikler T. Halitosis: The multidisciplinary approach. Int J Oral Sci 2012;4:55-63.

10. Yokoyama S, Ohnuki M, Shinada K, Ueno M, Wright FA, Kawaguchi Y. Oral malodor and related factors in Japanese senior high school students. J Sch Health 2010;80:346-52.

11. Campisi G, Musciotto A, Di Fede O, Di Marco V, Craxì A. Halitosis: Could it be more than mere bad breath? Intern Emerg Med 2011;6:315-9.

12. Kim SY, Sim S, Kim SG, Park B, Choi HG. Prevalence and associated factors of subjective halitosis in Korean adolescents. PLoS One 2015;10:e0140214.

13. Bornstein MM, Kislig K, Hoti BB, Seemann R, Lussi A. Prevalence of halitosis in the population of the city of Bern, Switzerland: A study comparing self-reported and clinical data. Eur J Oral Sci 2009;117:261-7.

14. Aimetti M, Perotto S, Castiglione A, Ercoli E, Romano F. Prevalence estimation of halitosis and its association with oral health-related parameters in an adult population of a city in North Italy. J Clin Periodontol 2015;42:1105-14.

15. AlSadhan SA. Self-perceived halitosis and related factors among adults residing in Riyadh, Saudi Arabia. A cross sectional study. Saudi Dent J 2016;28:118-23.

16. Nalcaci R, Baran I. Factors associated with self-reported halitosis (SRH) and perceived taste disturbance (PTD) in elderly. Arch Gerontol Geriatr 2008;46:307-16.

17. Eli I, Baht R, Koriat H, Rosenberg M. Self-perception of breath odor. J Am Dent Assoc 2001;132:621-6.

18. Rayman S, Almas K. Halitosis among racially diverse populations: An update. Int J Dent Hyg 2008;6:2-7.

19. Calil CM, Marcondes FK. Influence of anxiety on the production of oral volatile sulfur compounds. Life Sci 2006;79:660-4.

20. Settineri S, Mento C, Gugliotta SC, Saitta A, Terranova A, Trimarchi G, et al. Self-reported halitosis and emotional state: Impact on oral conditions and treatments. Health Qual Life Outcomes 2010;8:34.

21. Suzuki N, Yoneda M, Naito T, Iwamoto T, Hirofuji T. Relationship between halitosis and psychologic status. Oral Surg Oral Med Oral Pathol Oral Radiol Endod 2008;106:542-7.

22. Rani H, Ueno M, Zaitsu T, Furukawa S, Kawaguchi Y. Factors associated with clinical and perceived oral malodor among dental students. J Med Dent Sci 2015;62:33-41.

23. Setia S, Pannu P, Gambhir RS, Galhotra V, Ahluwalia P, Sofat A. Correlation of oral hygiene practices, smoking and oral health conditions with self perceived halitosis amongst undergraduate dental students. J Nat Sci Biol Med 2014;5:67-72.

24. Ashwath B, Vijayalakshmi R, Malini S. Self-perceived halitosis and oral hygiene habits among undergraduate dental students. J Indian Soc Periodontol 2014;18:357-60.

25. Almas K, Al-Hawish A, Al-Khamis W. Oral hygiene practices, smoking habit, and self-perceived oral malodor among dental students. J Contemp Dent Pract 2003;4:77-90.

26. Bhat MY, Alayyash AA. Social stigma related to halitosis in Saudi and British population: A comparative study. J Dent Res Rev 2016;3:65. 
27. Evirgen S, Kamburoglu K. Effects of tongue coating and oral health on halitosis among dental students. Oral Health Prev Dent 2013;11:169-73.

28. Seemann R, Bizhang M, Djamchidi C, Kage A, Nachnani S. The proportion of pseudo-halitosis patients in a multidisciplinary breath malodour consultation. Int Dent J 2006;56:77-81.

29. Liu XN, Shinada K, Chen XC, Zhang BX, Yaegaki K, Kawaguchi Y. Oral malodor-related parameters in the Chinese general population. J Clin Periodontol 2006;33:31-6.

30. Quirynen M, Dadamio J, Van den Velde S, De Smit M, Dekeyser C, Van Tornout M, et al. Characteristics of 2000 patients who visited a halitosis clinic. J Clin Periodontol 2009;36:970-5.

31. Youngnak-Piboonratanakit P, Vachirarojpisan T. Prevalence of self-perceived oral malodor in a group of thai dental patients.
J Dent (Tehran) 2010;7:196-204.

32. Faveri M, Hayacibara MF, Pupio GC, Cury JA, Tsuzuki CO, Hayacibara RM. A cross-over study on the effect of various therapeutic approaches to morning breath odour. J Clin Periodontol 2006;33:555-60.

33. Figueiredo LC, Rosetti EP, Marcantonio E Jr., Marcantonio RA, Salvador SL. The relationship of oral malodor in patients with or without periodontal disease. J Periodontol 2002;73:1338-42.

34. Snel J, Burgering M, Smit B, Noordman W, Tangerman A, Winkel EG, et al. Volatile sulphur compounds in morning breath of human volunteers. Arch Oral Biol 2011;56:29-34.

35. Levin KA. Study design III: Cross-sectional studies. Evid Based Dent 2006;7:24-5. 